\title{
Utility of Endoscopic Ultrasound-Guided Fine Needle Aspiration in the Diagnosis of Local Recurrence of Pancreaticobiliary Cancer after Surgical Resection
}

Kazuyuki Matsumoto, Hironari Kato, Shigeru Horiguchi, Takeshi Tomoda, Akihiro Matsumi, Yuki Ishihara, Yosuke Saragai, Saimon Takada, Shinichiro Muro, Daisuke Uchida, and Hiroyuki Okada

Department of Gastroenterology and Hepatology, Okayama University Graduate School of Medicine, Dentistry and Pharmaceutical Science, Okayama, Japan

Background/Aims: Endoscopic ultrasound (EUS)-guided fine needle aspiration (FNA; EUS-FNA) allows for diagnostic tissue specimens from various regions to be analyzed. However, diagnosing recurrent pancreaticobiliary cancer after surgery is sometimes difficult. We evaluated the efficacy of EUS-FNA in the diagnosis of local recurrence of pancreaticobiliary cancer and analyzed the factors associated with falsenegative results. Methods: Fifty-one consecutive patients who underwent EUS-FNA due to suspected recurrence of pancreaticobiliary cancer after surgery in an academic center were retrospectively analyzed. The criteria for EUS-FNA were a resected margin or remnant pancreas mass, round swollen lymph node ( $\geq 10 \mathrm{~mm}$ in diameter), and soft-tissue enhancement around a major artery. Patients with suspected liver metastasis or malignant ascites were excluded. Results: Thirty-nine of the 51 patients had pancreatic cancer; the remaining 12 had biliary cancer. The target sites for EUS-FNA were the soft tissue around a major artery $(n=22,43 \%)$, the resected margin or remnant pancreas $(n=12,24 \%)$, and the lymph nodes ( $n=17,33 \%)$. The median size of the suspected recurrent lesions was $15 \mathrm{~mm}$ (range, 8 to $40 \mathrm{~mm}$ ). The overall sensitivity, specificity and accuracy of EUS-FNA for the diagnosis of recurrence was 84\% (32/38), 100\% (13/13), and $88 \%$ (45/51), respectively. FNA of the soft tissue around major arteries (odds ratio, 8.23; 95\% confidence interval, 1.2 to $166.7 ; p=0.033$ ) was significantly associated with a falsenegative diagnosis in the multivariate analysis. Conclusions: EUS-FNA is useful for diagnosing recurrent cancer, even after pancreaticobiliary surgery. The diagnoses of recurrence at soft-tissue sites should be interpreted with caution. (Gut
Liver 2020;14:652-658)

Key Words: Endoscopic ultrasound-guided fine needle aspiration; Pancreaticobiliary cancer; Neoplasm recurrence

\section{INTRODUCTION}

The prognosis of pancreaticobiliary cancer is one of the poorest among all malignancies. The 5-year survival rates among all patients with pancreatic or biliary cancer are less than $6 \%$ and $14 \%$, respectively. ${ }^{1}$ Complete surgical resection is the only curative treatment for this disease and is an independent predictor of postoperative survival. However, even after resection with a negative margin, the vast majority of cancers recur, either as distant metastases or local disease. ${ }^{2-7}$

Endoscopic ultrasound (EUS)-guided fine needle aspiration (FNA; EUS-FNA) is a very sensitive tool for the initial diagnosis of pancreaticobiliary cancer. The advantage of EUS-FNA in the diagnosis of pancreaticobiliary disease is the ability to perform percutaneous biopsy from a region that is often otherwise inaccessible to provide a tissue diagnosis. The sensitivity and specificity of EUS-FNA in the diagnosis of pancreaticobiliary cancer are reported to be $85 \%$ and $98 \%$, respectively, for pancreatic cancer, ${ }^{8}$ and $91 \%$ and $89 \%$ for biliary cancer., ${ }^{9,10}$ However, the accuracy of an EUS-FNA in the diagnosis of recurrent cancer is lower than that at its initial diagnosis. ${ }^{7}$ The problems of EUSFNA after pancreaticobiliary surgery (e.g., pancreaticoduodenectomy; PD), include difficulty in visualizing the target lesion and securing a safe puncture route. Thus, the aspirated specimen is sometimes insufficient for a definite pathological diagnosis.

\footnotetext{
Correspondence to: Kazuyuki Matsumoto

Department of Gastroenterology and Hepatology, Okayama University Graduate School of Medicine, Dentistry and Pharmaceutical Science, 2-5-1 Shikata-cho, Okayama 700-8558, Japan

Tel: +81-86-225-5991, Fax: +81-86-235-7219, E-mail: matsumotokazuyuki0227@yahoo.co.jp

Received on June 8, 2019. Revised on August 17, 2019. Accepted on September 9, 2019. Published online December $12,2019$. pISSN 1976-2283 eISSN 2005-1212 https://doi.org/10.5009/gnl19200

(a) This is an Open Access article distributed under the terms of the Creative Commons Attribution Non-Commercial License (http://creativecommons.org/licenses/by-nc/4.0) which permits unrestricted non-commercial use, distribution, and reproduction in any medium, provided the original work is properly cited.
} 
Furthermore, pancreaticobiliary cancer sometimes recurs in the nerve plexus around a major artery.

To the best of the authors' knowledge, there has only been one report about EUS-FNA for local recurrence after pancreatic cancer surgery, ${ }^{7}$ and there have been no reports about EUS-FNA for the diagnosis of recurrent cancer after pancreaticobiliary surgery in a large study population. Considering the efficacy of recent chemotherapy for pancreaticobiliary cancer, it is useful to diagnose recurrent cancer at an early stage. In this study, we evaluated the usefulness of EUS-FNA in the diagnosis of suspected cancer recurrence after pancreaticobiliary surgery, and also analyzed the risk factors for a false-negative diagnosis.

\section{MATERIALS AND METHODS}

\section{Patients}

The criteria for performing EUS-FNA were as follows: the appearance of a mass in the resected margin or remnant pancreas, a round swollen lymph node with a diameter of $\geq 10 \mathrm{~mm}$, and enhancement of the soft tissue around a major artery (e.g., celiac artery, superior mesenteric artery, and common hepatic artery) (Figs 1-3). Patients with suspected liver metastasis or malignant ascites or recurrence at the resected margin after R1 resection were excluded. During the study period (January 2006 to March 2018), 57 patients were considered for EUS-FNA due to suspected tumor recurrence after attempted curative resection for pancreaticobiliary cancer at our institution. Among them, the target lesion could not be observed in one patient, and EUSFNA was technically difficult to perform in three patients (due to an interruption of the vessels, target: the presence of soft tissue around the superior mesenteric artery: 2, and the occurrence of a lymph nodule around aorta: 1). The technical success rate of EUS-FNA was 93\% (53/57). Of the 53 patients who underwent EUS-FNA successfully, two experienced recurrence at the
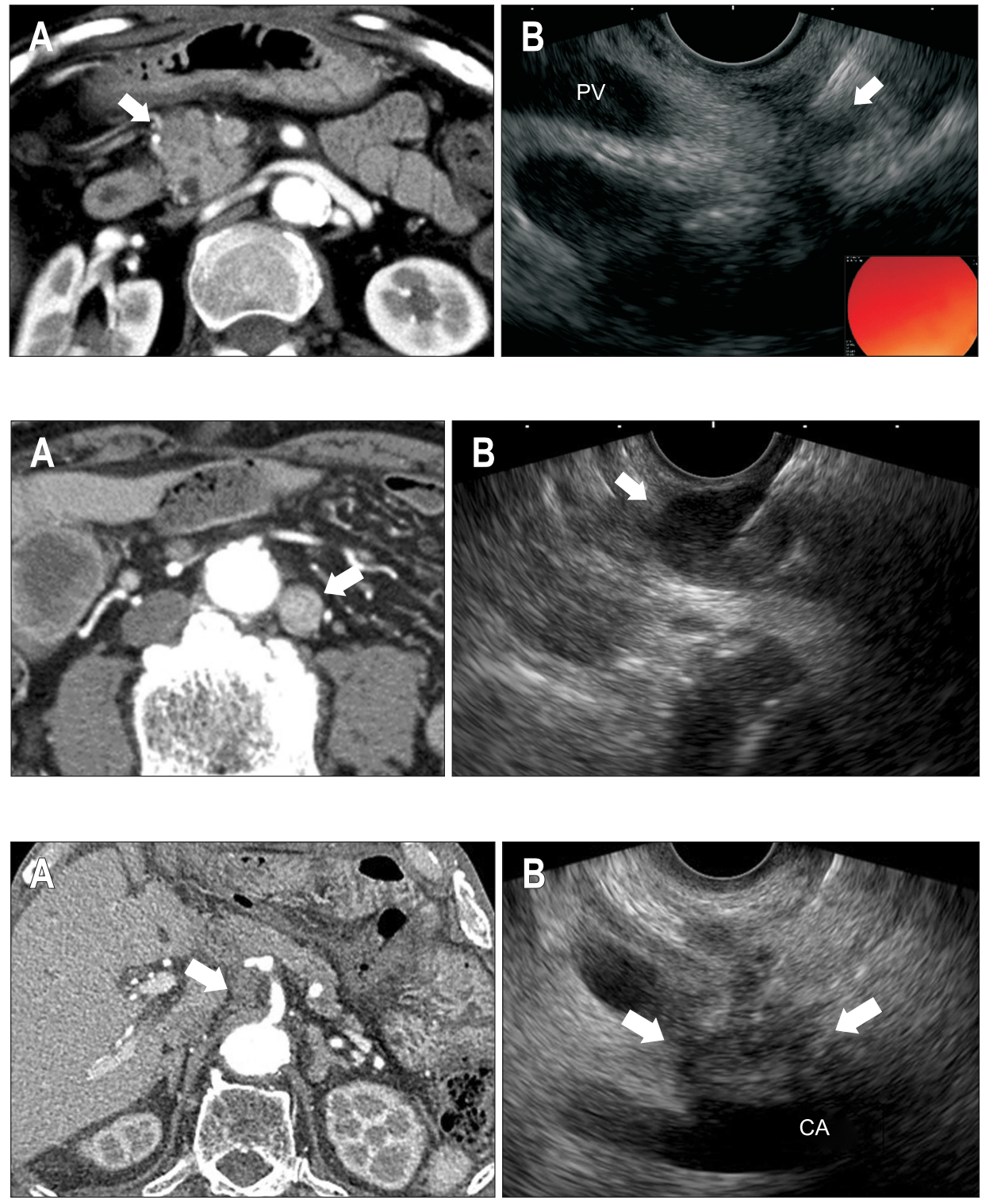

Fig. 3. (A) Computed tomography showing soft tissue around a major artery (arrow). (B) Endoscopic ultrasound-guided fine needle aspiration of soft tissue (arrows).

CA, celiac artery. 
resected margin after R1 resection. Ultimately, 51 patients were retrospectively analyzed in this study (Fig. 4).

One of the following surgical procedures was applied in all cases: classical PD, subtotal stomach-preserving pancreaticoduodenectomy, distal pancreatectomy, total pancreatectomy, distal pancreatectomy with en bloc celiac axis resection, hepatic resection and hepaticojejunostomy, or hepatopancreaticoduodenectomy. The resected cancers were pathologically classified according to the International Union against Cancer TNM Classification of Malignant Tumors 6th edition.

This study was approved by our institution's review board for human research and was conducted in accordance with the Declaration of Helsinki (Okayama University Graduate School of Medicine, Dentistry and Pharmaceutical Science and Okayama University Hospital, Ethics Committee, approval number: 1805024). Written informed consent was obtained from all subjects.

\section{EUS-FNA techniques}

We performed EUS using a convex linear-array endoscope (GF-UCT260 or GF-UCT240; Olympus Medical Systems, Tokyo, Japan and EG-580UT: Fujifilm Corp, Tokyo, Japan) connected to an ultrasound device (Prosound SSD- $\alpha 10$; Hitachi Aloka, Tokyo, Japan, EU-ME1 or EU-ME2 PREMIER PLUS; Olympus, SU-1 ultrasound processor; Fujifilm Corp). EUS-FNA was performed with a 22- and/or 25-gauge needle (EZ-Shot 3; Olympus Medical Systems; Acquire, Boston Scientific Corp., Marlborough, MA, USA; EchoTip ProCore, Cook Medical, Bloomington, IN, USA; SonoTip II, Medi-Globe GmbH, Rohrdorf, Germany; Expect, Boston Scientific Corp.).

EUS-FNA was repeated until sufficient sampling had been performed for rapid on-site evaluation or until the endoscopist believed that further sampling was unlikely to increase the amount of tissue.

\section{The pathological diagnosis}

All aspirated samples were expressed onto a glass slide by reinsertion of the stylet into the FNA needle. The aspirated samples were divided into a white tissue part for histological evaluation and the remaining portion was used for a cytological evaluation. Two smear preparations of the samples were made for each cytological evaluation: One slide was subjected to modified Giemsa staining for rapid on-site evaluation, whereas the other slide was alcohol-fixed and subjected to Papanicolaou staining. A definitive cytohistopathological diagnosis was made by two pathologists. For EUS-FNA samples, specimens were classified as having no malignancy, atypical cells, malignancy, or being inadequate if representative material was not present When pathological specimens did not disclose any definitive malignancy or were inadequate, clinical and/or imaging followup examinations were performed every 2 to 3 months, and the definitive diagnosis of benign was decided after at least 6 months of follow-up.

\section{Statistical analyses}

Continuous variables were expressed as the median and range, or interquartile range (IQR). Categorical variables were analyzed using the chi-square test. The Student t-test was used to compare continuous variables. Factors with p-values $<0.20$ using the forward method were considered to be potential risk factors for a false-negative diagnosis and were analyzed in a multiple logistic regression model. Odds ratios and 95\% confidence intervals were calculated. Two-tailed p-values $<0.05$ were considered to indicate statistical significance. All analyses were performed using the JMP Pro 12 software program (SAS Institute, Cary, NC, USA).

\section{RESULTS}

\section{Patient characteristics}

Table 1 summarizes the characteristics of 51 patients who underwent EUS-FNA due to suspected recurrence of pancreaticobiliary cancer after surgical resection. The median age of the patients was 66 years (range, 50 to 84 years). The primary diseases were pancreatic cancer in 39 patients (76\%) and biliary cancer in 12 patients (24\%). A total of 47 and four patients underwent R0 and R1 resection, respectively. Forty-six patients (90\%) received adjuvant therapy with S-1, and no patients received radiation therapy after surgery. The median value of

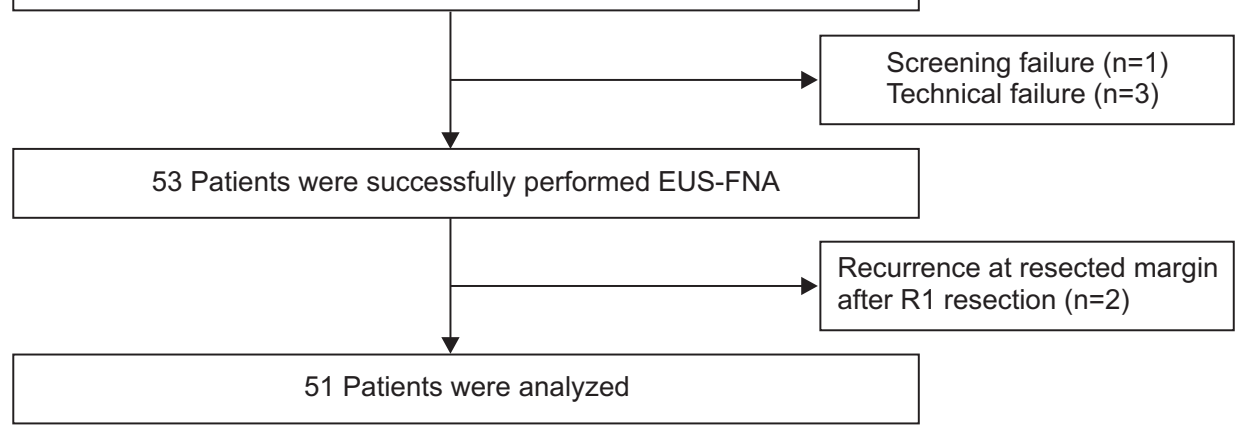

Fig. 4. The flowchart of this study. EUS-FNA, endoscopic ultrasoundguided fine needle aspiration. 
serum carbohydrate antigen 19-9 (CA19-9) at EUS-FNA was $141 \mathrm{U} / \mathrm{mL}$ (IQR, 22 to $303 \mathrm{U} / \mathrm{mL}$ ), and 26 patients (51\%) showed an abnormal level of CA19-9. The median interval between the operation and EUS-FNA was 13.0 months (IQR, 7.3 to 31.9 months). The median flow-up period after EUS-FNA for sites of recurrence and the initial diagnosis were 16.1 months (IQR, 7.9 to 25.5 months) and 34.7 months (IQR, 22.3 to 56.7 months).

\section{EUS-FNA for suspected recurrent lesions}

Table 2 shows the results of EUS-FNA for 51 patients with suspected recurrence. The median size of the suspected recurrent lesion, excluding the soft tissue around a major artery, was $15 \mathrm{~mm}$ (range, 8 to $40 \mathrm{~mm}$ ). The target site for EUS-FNA was the soft tissue around a major artery in 22 patients (43\%), the resected margin in seven patients (14\%), the remnant pancreas in five patients (10\%), and the lymph nodes in 17 patients (33\%). Among patients who underwent EUS-FNA at the soft tissue around the artery, six (27\%) showed an unclear low-echoic le-

Table 1. Patient Characteristics $(\mathrm{n}=51)$

\begin{tabular}{lc}
\hline \multicolumn{1}{c}{ Parameter } & Value \\
\hline Age, median (range), yr & $66(50-84)$ \\
Male sex & $28(55)$ \\
Diseases & \\
Pancreatic cancer & $39(76)$ \\
Biliary cancer & $12(24)$ \\
$\quad$ BDCa/GBCa/PVCa & $10 / 1 / 1$ \\
Surgical method & \\
SSPPD/PD & $28(55)$ \\
DP & $12(25)$ \\
DP-CAR & $3(5)$ \\
TP & $1(2)$ \\
HR+HJ & $5(9)$ \\
HPD & $2(4)$ \\
Residual tumor & \\
R0/1 & $47(92) / 4(8)$ \\
Adjuvant chemotherapy & $46(90)$ \\
Serum CA19-9 at EUS-FNA, U/mL & $141(22-303)$ \\
Patients with CA19-9 value $>40 ~ U / m L$ & $26(51)$ \\
Interval between operation and EUS-FNA, mo & $13.0(7.3-31.9)$ \\
Follow-up period after EUS-FNA, mo & $16.1(7.9-25.5)$ \\
Follow-up after initial diagnosis, mo & $34.7(22.3-56.7)$ \\
\hline
\end{tabular}

Data are presented as number (\%) or median (interquartile range). BDCa, bile duct cancer; GBCa, gall bladder cancer; PVCa, papilla of Vater cancer; SSPPD, subtotal stomach-preserving pancreaticoduodenectomy; PD, pancreaticoduodenectomy; DP, distal pancreatectomy; DP-CAR, distal pancreatectomy with en bloc celiac axis resection; $\mathrm{TP}$, total pancreatectomy; HR, hepatic resection; HJ, hepaticojejunostomy; HPD, hepatopancreaticoduodenectomy; CA19-9, carbohydrate antigen 19-9; EUS-FNA, endoscopic ultrasound-guided fine needle aspiration. sion on EUS imaging. The median number of needle passes was 2 (range, 1 to 7). The needle size used for FNA was 22-gauge in 36 cases (70\%) and 25-gauge in 15 cases (30\%).

\section{The pathological diagnosis of EUS-FNA samples}

For 51 patients, the final diagnosis after clinical follow-up was recurrent pancreaticobiliary cancer in 38 cases and nonrecurrence of pancreaticobiliary cancer in 13 cases. Of the 38 samples from recurrent cases, 32 samples were positive for malignancy and six were benign (atypical cells, $n=2$; no malignancy, $n=2$; inadequate, $n=2$ ). Of the six patients in which the pathological diagnosis was benign, three patients underwent EUS-FNA of the same lesion again within 3 months, and all samples were pathologically malignant (soft tissue, $n=2$; lymph node, $n=1$ ). The other three patients developed recurrent disease as metastasis to the liver and peritoneum on computed tomography (CT) during clinical follow-up (soft tissue, $n=3$ ). In comparison, among the 13 non-recurrent samples, six samples were non-malignant, three samples were atypical, and four were inadequate. No patients developed recurrent disease during the follow-up period after EUS-FNA (median, 25.3 months; IQR, 15.2 to 47.8 months).

\section{Diagnostic ability of EUS-FNA}

The sensitivity, specificity, and overall accuracy were 84\% (32/38), 100\% (13/13), and 88\% (45/51), respectively. According to the disease, the sensitivity, specificity and overall accuracy were $86 \%$ (25/29), 100\% (10/10), and 90\% (35/39) in pancreatic cancer and 78\% (7/9), 100\% (3/3), and 83\% (10/12) in biliary cancer, respectively. According to the site of the EUS-FNA, the sensitivity, and accuracy were as follows: soft tissue around a

Table 2. EUS-FNA for Suspected Recurrent Lesions ( $\mathrm{n}=51)$

\begin{tabular}{lc}
\hline \multicolumn{1}{c}{ Parameter } & Value \\
\hline Size of suspected recurrent lesion, mm* & $15(8-40)$ \\
FNA site of suspected recurrence & \\
Soft tissue around artery & $22(43)$ \\
CA/SMA/CHA & $10 / 7 / 5$ \\
Resected margin & $7(14)$ \\
Remnant pancreas & $5(10)$ \\
Lymph node & $17(33)$ \\
Around aorta/stomach/portal vein & $7 / 6 / 4$ \\
No. of needle passes & $2(1-7)$ \\
Selected FNA needle gauge & \\
22 & $36(70)$ \\
25 & $15(30)$ \\
\hline
\end{tabular}

Data are presented as median (range) or number (\%).

EUS-FNA, endoscopic ultrasound-guided fine needle aspiration; CA, celiac artery; SMA, superior mesenteric artery; CHA, common hepatic artery.

*Soft-tissue lesions excluded. 
major artery: 55\% (6/11) and 77\% (17/22); remnant or margin of the pancreas: 100\% (12/12) and 100\% (12/12); and lymph node: 93\% (14/15) and 94\% (16/17), respectively (Table 3). In the analysis of the soft tissue around the artery, the accuracy in patients with clear and unclear low-echoic masses were $81 \%$ $(13 / 16)$ and $67 \%(4 / 6)$, respectively $(\mathrm{p}=0.47)$.

\section{Analyses of the risk factors for a false-negative diagnosis}

In the analysis of the risk factors for a false-negative diagnosis, surgery with stomach resection, and soft tissue around a major artery were identified as potential factors $(p<0.2)$ in univariate analyses. Multiple logistic regression revealed that soft tissue around a major artery (odds ratio, 8.23; 95\% confidence interval, 1.2 to $166.7 ; p=0.033$ ) was a significant risk factor for a false-negative diagnosis (Table 4).

\section{DISCUSSION}

This study was the first report to evaluate the diagnostic ability of EUS-FNA in the detection of recurrence after pancreaticobiliary surgery in a large cohort and with a sufficient follow- up period (median flow-up period after EUS-FNA for recurrence sites was 16.1 months). The overall sensitivity, specificity and accuracy of EUS-FNA in the diagnosis of recurrence were 84\% (32/38), 100\% (13/13), and 88\% (45/51), respectively. EUS-FNA is useful for diagnosing the recurrence of cancer, even after pancreaticobiliary surgery. We also analyzed the factors associated with a false-negative diagnosis, and FNA of the soft tissue around major arteries (odds ratio, 8.23; 95\% confidence interval, 1.2 to $166.7 ; p=0.033$ ) was found to be significantly associated with false-negative diagnoses in the multivariate analysis.

DeWitt et al. ${ }^{7}$ reported the usefulness of EUS-FNA in the diagnosis of 17 patients with suspected recurrence of pancreatic cancer: The sensitivity and accuracy of EUS-FNA ranged from $81 \%$ to $93 \%$ and $81 \%$ to $93 \%$, respectively. In our study, the overall sensitivity, and accuracy of EUS-FNA in the diagnosis of recurrence were 84\% (32/38), and 88\% (45/51), respectively. For limited pancreatic cancer recurrence, the sensitivity and accuracy of EUS-FNA in this study were $86 \%$ and $90 \%$, respectively. Our results proved similar to those of past reports. EUS-FNA is useful for diagnosing recurrence, even after pancreaticobiliary surgery.

Table 3. Performance of EUS-FNA for the Diagnosis of Cancer Recurrence $(n=51)$

\begin{tabular}{|c|c|c|c|c|c|}
\hline & Sensitivity, $\%$ & Specificity, \% & PPV, \% & NPV, \% & Accuracy, \% \\
\hline Total & 84 & 100 & 100 & 68 & 88 \\
\hline Pancreatic cancer & 86 & 100 & 100 & 71 & 90 \\
\hline Biliary cancer & 78 & 100 & 100 & 60 & 83 \\
\hline \multicolumn{6}{|l|}{ According to recurrence site } \\
\hline Soft tissue around major artery & 55 & 100 & 100 & 69 & 77 \\
\hline Resected margin/remnant pancreas & 100 & NA & 100 & NA & 100 \\
\hline Lymph nodule & 93 & 100 & 100 & 67 & 94 \\
\hline
\end{tabular}

EUS-FNA, endoscopic ultrasound-guided fine needle aspiration; PPV, positive predictive value; NPV, negative predictive value; NA, not available.

Table 4. Univariate and Multivariate Analyses of Factors Associated with False-Negative Results

\begin{tabular}{|c|c|c|c|c|c|}
\hline \multirow{2}{*}{ Variable } & \multirow{2}{*}{$\begin{array}{l}\text { No. false negatives } \\
\qquad(\mathrm{n}=6)\end{array}$} & \multirow{2}{*}{$\begin{array}{l}\text { No. true positives } \\
\qquad(\mathrm{n}=45)\end{array}$} & \multirow{2}{*}{$\begin{array}{c}\text { Univariate analysis } \\
\text { p-value }\end{array}$} & \multicolumn{2}{|c|}{ Multivariate analysis } \\
\hline & & & & OR $(95 \% \mathrm{CI})$ & $\mathrm{p}$-value \\
\hline Age $>65$ yr & 3 & 23 & 0.96 & - & - \\
\hline Male sex & 4 & 24 & 0.53 & - & - \\
\hline Biliary cancer & 2 & 10 & 0.56 & - & - \\
\hline CA19-9 >40 U/mL & 2 & 24 & 0.35 & - & - \\
\hline Adjuvant chemotherapy & 6 & 40 & 0.25 & - & - \\
\hline Surgery with stomach resection* & 5 & 26 & 0.2 & $3.65(0.48-75.6)$ & 0.22 \\
\hline Soft-tissue around major artery & 5 & 17 & 0.03 & $8.23(1.2-166.7)$ & 0.033 \\
\hline Use of a 22-gauge needle & 4 & 32 & 0.82 & - & - \\
\hline No. of needle passes >2 & 4 & 18 & 0.21 & - & - \\
\hline
\end{tabular}

OR, odds ratio; CI, confidence interval; CA19-9, carbohydrate antigen 19-9.

*Including pancreaticoduodenectomy, subtotal stomach-preserving pancreaticoduodenectomy, total pancreatectomy, and hepatopancreaticoduodenectomy. 
The sensitivity and accuracy, according to the site of EUSFNA, was as follows: the soft tissue around a major artery: 55\% (6/11) and 77\% (17/22); remnant or margin of the pancreas: 100\% (12/12) and 100\% (12/12); and lymph node: 93\% (14/15) and 94\% (16/17), respectively. The diagnostic ability of EUSFNA was considered sufficient for recurrent disease at the pancreatic remnant or margin or lymph nodes. However, the diagnosis of soft tissue recurrence around major arteries was a problem. The median number of needle passes at soft tissue sites was 3 (rage, 1-7) times, which was significantly greater in comparison to other sites (2 [rage, 1-5] times, $\mathrm{p}<0.01$ ). A 22-gauge needle was used in 73\% (16/22) of the procedures for soft tissue and $69 \%(20 / 29)$ of the procedures at other sites ( $p=0.78)$. However, the accuracy of the results of EUS-FNA in the diagnosis of recurrence in the soft tissue around a major artery was lower than that at other sites. In our study, there were 16 (73\%) and six (27\%) patients with and without a low-echoic mass around the artery on EUS imaging. The accuracy of patients with clear and unclear low-echoic masses were 81\% (13/16) and 67\% (4/6), respectively $(\mathrm{p}=0.47)$. With such a small number of analyses, it might be difficult to predict recurrence using EUS images. This is a particular problem of EUS-FNA in the diagnosis of recurrent cancer. There are several reasons for this result. First, the visualization of the soft-tissue area around an artery via EUS is sometimes difficult. Second, the stroke range of the FNA needles was not sufficient because of tangled vessels, especially around the celiac artery. Third, cancer cells can become sparsely distributed into the interstices around an artery without forming a mass. Thus, to increase the potential for a diagnosis, a different approach should be considered for the examination of EUSFNA specimens, such as KRAS gene mutation analysis. ${ }^{11,12}$

Contrast-enhanced CT (CE-CT) and positron emission tomography (PET)/CT are currently the most well-established and useful tools for restaging patients with suspicion of cancer recurrence. Kitajima et al. ${ }^{13}$ reported the diagnostic abilities of CECT and PET/CT for recurrence of pancreatic cancer after surgical resection. Forty-five patients, including those with radiological imaging follow-up, were analyzed, and the sensitivity, specificity, and accuracy of CE-CT were 66.7\% (16/24), 85.7\% (18/21), and $75.6 \%$ (34/45), respectively, whereas those of PET/CT were $83.3 \%$ (20/24), 90.5\% (19/21), and 86.7\% (39/45), respectively. In the 21 patients for whom the final diagnosis was obtained from the histopathologic examination, the sensitivity, specificity, and accuracy of CE-CT were 73.7\% (14/19), 50\% (1/2), and 71.4\% (15/21), respectively, while those of PET/CT were 89.5\% (17/19), 50\% (1/2), and 85.7\% (18/21), respectively. In our study, the sensitivity, specificity, and accuracy of EUS-FNA were 84\% (32/38), 100\% (13/13), and 88\% (45/51), respectively, which are considered sufficient when compared with CE-CT and PET/CT, especially in terms of the specificity. There were no false-positive cases with EUS-FNA, which is clinically important when assessing the recurrence of cancer after surgery.

For lesion site-based analyses, the sensitivities of CE-CT and PET/CT for abdominal lymph node were $62.5 \%$ and $75 \%$, and those for local recurrence were 50\% and 66.7\%, respectively. In our study, the sensitivity of EUS-FNA for lymph nodes was 93\% (16/17), while that for local recurrence (soft tissue and remnant or margin of the pancreas) was 85\% (29/34). The sensitivities of EUS-FNA for lymph nodes and local recurrence were sufficient compared with CE-CT and PET/CT. PET/CT was limited by the need for blood glucose levels to be checked strictly before the examination. There is a markedly high incidence of new-onset or worsening of diabetes mellitus after pancreatic resection. ${ }^{14}$ Magnetic resonance imaging is also useful for detecting local recurrence and liver metastasis. Magnetic resonance imaging showed a higher sensitivity than PET/CT in the detection of liver metastasis; however, its sensitivity for locoregional cancer recurrence was low. ${ }^{15}$

The level of CA19-9 was also used for diagnosing recurrence after pancreaticobiliary cancer. We examined the levels of CA19-9 at FNA, and the median value was $141 \mathrm{U} / \mathrm{mL}$. Of the 38 patients with recurrence, only 21 (55\%) showed abnormal levels of CA19-9. In contrast, of the 13 patients with non-recurrence, five $(38 \%)$ showed abnormal levels of CA19-9 $(p=0.30)$. It is therefore difficult to diagnose cancer recurrence using the CA19-9 level. EUS-FNA is not necessary in patients with obvious recurrence, such as liver metastasis or malignant ascites, so we excluded these patients. When we are unable to make a diagnosis with confidence based on radiographic images, we can obtain a definitive diagnosis of recurrence using EUS-FNA, allowing treatment for recurrence to be started at an early stage.

This study was associated with some limitations. First, it employed a retrospective, single center design, and the number of patients with recurrent biliary cancer was small. Second, the procedures were only performed when we considered EUS-FNA feasible based on radiographic images. Thus, the diagnostic ability, including the success rate of EUS-FNA, was not evaluated correctly. However, 92\% (23/25) of cases with soft tissue around the artery, which was considered difficult to puncture due to its location near a main artery or the existence of interrupted vessels or organs along the puncture route, successfully underwent EUS-FNA. Third, several types and sizes of needle were used for EUS-FNA, and procedures were performed by several examiners.

In conclusion, we evaluated the diagnostic ability of EUSFNA in cases in which recurrent pancreaticobiliary cancer was suspected after surgical resection. EUS-FNA is useful for diagnosing recurrent cancer, even after pancreaticobiliary surgery. However, FNA of the soft tissue around major arteries was significantly associated with false-negative results. The results of FNA of the soft tissue around major arteries should therefore be interpreted with caution, and close clinical follow-up is needed. 


\section{CONFLICTS OF INTEREST}

No potential conflict of interest relevant to this article was reported.

\section{AUTHOR CONTRIBUTIONS}

Conception and design of the research, and writing the paper: K.M., H.K. Analysis and interpretation of data: A.M., Y.I., S.T. Critical revision of the article for important intellectual content: S.H., Y.S., S.M., D.U., T.T. Final approval of the article: H.O. All authors read and approved the final manuscript.

\section{ORCID}

Kazuyuki Matsumoto https://orcid.org/0000-0002-5102-7452

Hironari Kato https://orcid.org/0000-0003-1120-292X

Shigeru Horiguchi https://orcid.org/0000-0001-9769-8262

Takeshi Tomoda https://orcid.org/0000-0002-5525-2796 https://orcid.org/0000-0003-0923-6143 https://orcid.org/0000-0002-5515-1880 https://orcid.org/0000-0001-7710-2329 https://orcid.org/0000-0002-0957-6909 https://orcid.org/0000-0001-7626-4342 https://orcid.org/0000-0002-2809-2707 https://orcid.org/0000-0003-2814-7146

\section{REFERENCES}

1. Siegel R, Naishadham D, Jemal A. Cancer statistics, 2012. CA Cancer J Clin 2012;62:10-29.

2. Miyazaki M, Yoshitomi H, Shimizu H, et al. Repeat pancreatectomy for pancreatic ductal cancer recurrence in the remnant pancreas after initial pancreatectomy: is it worthwhile? Surgery 2014;155:58-66

3. Benassai G, Mastrorilli M, Quarto G, et al. Factors influencing survival after resection for ductal adenocarcinoma of the head of the pancreas. J Surg Oncol 2000;73:212-218.

4. Richter A, Niedergethmann M, Sturm JW, Lorenz D, Post S, Trede M. Long-term results of partial pancreaticoduodenectomy for ductal adenocarcinoma of the pancreatic head: 25-year experience.
World J Surg 2003;27:324-329.

5. Jarnagin WR, Ruo L, Little SA, et al. Patterns of initial disease recurrence after resection of gallbladder carcinoma and hilar cholangiocarcinoma: implications for adjuvant therapeutic strategies. Cancer 2003;98:1689-1700.

6. Koo TR, Eom KY, Kim IA, et al. Patterns of failure and prognostic factors in resected extrahepatic bile duct cancer: implication for adjuvant radiotherapy. Radiat Oncol J 2014;32:63-69.

7. DeWitt J, Sherman S, Al-Haddad M, McHenry L, Cote GA, Leblanc JK. EUS-guided FNA of local recurrence of pancreatic cancer after surgical resection. Gastrointest Endosc 2010;72:1076-1080.

8. Chang KJ, Nguyen P, Erickson RA, Durbin TE, Katz KD. The clinical utility of endoscopic ultrasound-guided fine-needle aspiration in the diagnosis and staging of pancreatic carcinoma. Gastrointest Endosc 1997;45:387-393.

9. Byrne MF, Gerke H, Mitchell RM, et al. Yield of endoscopic ultrasound-guided fine-needle aspiration of bile duct lesions. Endoscopy 2004;36:715-719.

10. Fritscher-Ravens A, Broering DC, Knoefel WT, et al. EUS-guided fine-needle aspiration of suspected hilar cholangiocarcinoma in potentially operable patients with negative brush cytology. Am J Gastroenterol 2004;99:45-51.

11. Fuccio L, Hassan C, Laterza L, et al. The role of K-ras gene mutation analysis in EUS-guided FNA cytology specimens for the differential diagnosis of pancreatic solid masses: a meta-analysis of prospective studies. Gastrointest Endosc 2013;78:596-608.

12. Sho S, Court CM, Kim S, et al. Digital PCR improves mutation analysis in pancreas fine needle aspiration biopsy specimens. PLoS One 2017;12:e170897.

13. Kitajima K, Murakami K, Yamasaki E, et al. Performance of integrated FDG-PET/contrast-enhanced CT in the diagnosis of recurrent pancreatic cancer: comparison with integrated FDG-PET/ non-contrast-enhanced CT and enhanced CT. Mol Imaging Biol 2010;12:452-459.

14. Burkhart RA, Gerber SM, Tholey RM, et al. Incidence and severity of pancreatogenic diabetes after pancreatic resection. J Gastrointest Surg 2015;19:217-225.

15. Javadi S, Karbasian N, Bhosale P, et al. Imaging findings of recurrent pancreatic cancer following resection. Abdom Radiol (NY) 2018;43:489-496. 